\title{
EVALUACIÓN DE LOS ESTILOS EDUCATIVOS PARENTALES EN UNA MUESTRA DE ESTUDIANTES FILIPINOS: IMPLICACIONES EDUCATIVAS
}

\author{
Alfonso Osorio de Rebellón Yohn, Sonia Rivas Borrell, Jokin de \\ Irala Estévez, María Calatrava, Cristina López del Burgo.
}
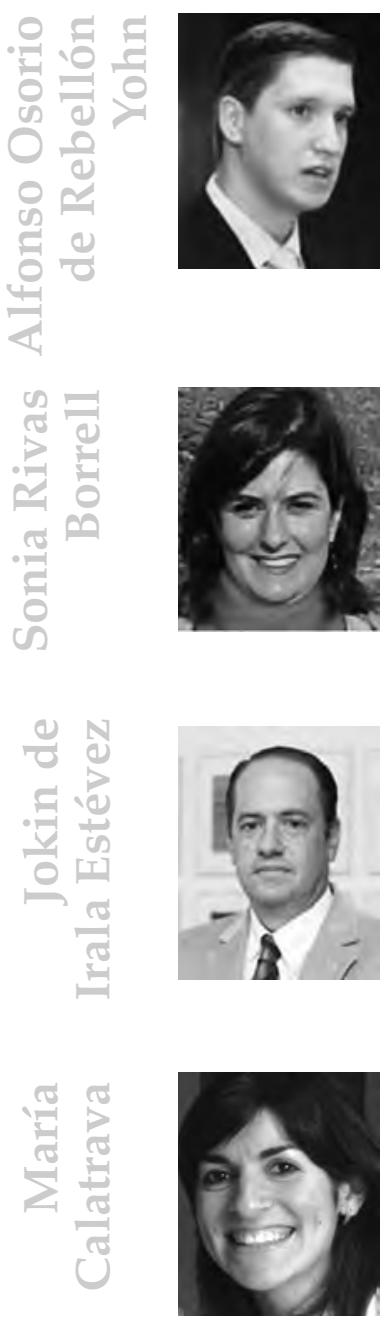

Licenciado y Doctor en Filosofía, Universidad de Navarra. Licenciado en Psicología, Universidad Nacional de Educación a Distancia, España. Profesor del Departamento de Educación de la Universidad de Navarra, y del Máster en Matrimonio y Familia de la misma universidad. Sus áreas de interés incluyen especialmente la educación de la juventud en el ámbito familiar.

Correo electrónico: [aosorio@unav.es].

Profesora ayudante doctora del Departamento de Educación, Universidad de Navarra. Profesora del Máster en Matrimonio y Familia, Universidad de Navarra. Sus líneas de investigación se centran en la evaluación del ámbito familiar y en los programas de educación infantil.

Correo electrónico: [srivas@unav.es].

Doctor en Epidemiología, Universidad de Massachusetts. Profesor titular de Epidemiología. Subdirector del Departamento de Medicina Preventiva y Salud Pública, Universidad de Navarra. Subdirector del Instituto de Ciencias para la Familia, Universidad de Navarra. Sus principales áreas de investigación son los temas de sexualidad y salud reproductiva.

Correo electrónico: [jdeirala@unav.es].

Investigadora de Medicina Preventiva, Universidad de Navarra. Su investigación se centra en la salud de los adolescentes y jóvenes, así como en los estilos de vida relacionados con la iniciación sexual y otras conductas de riesgo. Correo electrónico: [mcalatrava@alumni.unav.es]. 


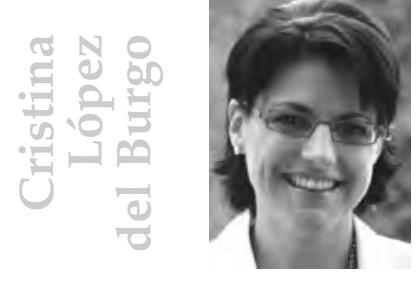

Doctora en Medicina, Universidad de Navarra y especialista en Medicina Familiar y Comunitaria. Trabaja actualmente como Profesora del Departamento de Medicina Preventiva y Salud Pública, Universidad de Navarra. Su investigación se centra en temas de salud sexual y reproductiva, especialmente en la planificación familiar y la epidemiología reproductiva.

Correo electrónico: [cdelburgo@unav.es].

A Intermedia Consulting, por haber promovido este proyecto. A Antonio N. Torralba y Vina L. Arenal, de la Universidad de Asia y el Pacífico (Manila), y a Filipinas O. de Guzman, de CRC Foundation Incorporated (Manila), por haber implementado el estudio en Filipinas.

\section{RESUMEN}

Un cuerpo teórico importante de investigación sugiere que el estilo autoritativo de los padres se asocia con unas mejoras en diversos aspectos de ajuste psicosocial de los adolescentes. Este artículo desarrolla y analiza la tipología de los estilos educativos parentales en el contexto de jóvenes filipinos. A partir de una muestra de 3.601 estudiantes, se muestra que el estilo autoritativo se asocia con mayor autoestima, menor impulsividad, mayor intención de alcanzar metas positivas en el futuro, mejor rendimiento académico, menor prevalencia de experiencia sexual y mayor rechazo de diversas formas de sexismo.

Palabras clave: estilos educativos; estilos parentales; jóvenes; adolescentes; ajuste psicosocial; Filipinas.

\section{ABSTRACT}

An important amount of research suggests that parents' authoritative style is associated with improvements in different aspects of adolescents' psycho-social adjustment. This article develops and analyzes the typology of parental educative styles, within the context of Filipino students. 
Using a sample of 3601 students, we found that authoritative style is associated to higher self-esteem, lower impulsivity, higher intention to fulfill positive future goals, better academic achievement, lower prevalence of sexual experience and higher rejection of different forms of sexism.

Key words: educative styles; parenting styles; youth; adolescents; psychosocial adjustment; Philippines.

\section{INTRODUCCIÓN}

Desde un punto de vista educativo, la familia constituye el marco informal idóneo para que los padres eduquen, transfiriendo sus conocimientos y estrategias a sus hijos, siendo las relaciones intrafamiliares su componente central. Sin embargo, son notables las diferencias en el funcionamiento familiar entre las distintas familias, entre unas y otras culturas, y a lo largo del tiempo; diferencias que afectan directamente a las relaciones intra-familiares, tanto en grado como en contenido. Si nos referimos a la realidad de los países asiáticos, comprobamos que cada grupo social y cada país suele caracterizarse por el desempeño de unos roles diferentes, que reflejan su diversidad a la vez que su singularidad. De todos modos, existen muchas similitudes y parece haber unanimidad en señalar el papel central que se le otorga a la familia (Wolf, 1997), la interdependencia entre sus miembros, su cohesión ${ }^{1}$, la unidad, el uso del control familiar y su sentido estricto en la educación en cuanto a la búsqueda del éxito escolar en los hijos. En contraste con las familias del Este asiático y con partes de la India, la realidad filipina parece ser una excepción (Peabody, 1968). Precisamente, dicha sociedad está siendo estudiada con el fin de determinar las semejanzas y diferencias con países de otras culturas y con la sociedad occidental, en cuanto a las relaciones que se establecen en su núcleo familiar (Fuligni, 1998; Huynh y Fuligni, 2008, Rousseau, Hassan, Measham, Moreau, Lashley, Castro, Blake y McKenzie, 2009).

1 Espriu y Wolf (2001) apuntan que la aversión que tiene la sociedad filipina hacia el divorcio puede explicar el alto porcentaje de familias nucleares, a pesar de los frecuentes informes sobre el conflicto familiar en el país. 


\section{UNA APROXIMACIÓN A LOS ESTILOS EDUCATIVOS}

El estudio del entorno familiar como agente socializador de los hijos y como marco idóneo de la transmisión de valores se ha analizado desde el conjunto de las relaciones y lazos creados entre sus miembros, específicamente entre los padres y los hijos. En este contexto es donde cabe referirse a estilos educativos paternos.

Entendemos por estilo educativo,

«los esquemas prácticos que reducen las múltiples y minuciosas pautas educativas paternas a unas pocas dimensiones básicas que, cruzadas entre sí, en diferentes combinaciones, dan lugar a diversos tipos habituales de educación familiar» (Coloma, 1993, p. 48).

Éstos constituyen el eje fundamental mediante el que puede analizarse la interacción paterno-filial, y en torno al cual se distribuye el contenido y se delimitan formas, estrategias, procedimientos y expectativas de la educación familiar.

El análisis y estudio de dichos estilos cuenta con una larga tradición en las ciencias sociales. Desde los primeros estudios que se remontan a los años 30 (Coloma, 1993), las investigaciones encontraron su apogeo en las últimas décadas del siglo XX.

En los años setenta comenzó el estudio de la familia desde una visión sociológica, en cuanto se reconocían las relaciones y las interacciones entre sus miembros (Bronfenbrenner, 1979; Sameroff y Chandler, 1975). Un considerable volumen de literatura científica abordó en tipologías los estilos de socialización, como un intento por integrar y aunar el modo en que se comportan los padres en relación con sus hijos. La mayoría de estas investigaciones partían del modelo clásico tripartito formulado por Diane Baumrind $(1968,1971)$, quien proponía el modelo democrático, autoritario y permisivo, a partir de la interrelación de tres variables paternas básicas: control, comunicación e implicación afectiva. 
En 1983, Maccoby y Martin reformularon el modelo anterior tomando en cuenta otra dimensión asociada al control de los padres: la contingencia del esfuerzo parental y el nivel de exigencia (Musitu, Román y García, 2001). Estos autores completaron la clasificación anterior refiriéndose al estilo paterno negligente o indiferente. El resultado de dichas aportaciones fueron los cuatro estilos educativos que, sin bien han sido reformulados por otros autores ${ }^{2}$, siguen manteniendo su vigencia: el estilo autoritativo ${ }^{3}$, también llamado democrático; el estilo negligente; el permisivo, y el estilo autoritario. El estilo autoritativo se define así por alto nivel en control y responsabilidad; el segundo, el estilo negligente, es propio de las familias con bajo nivel en control y en responsabilidad; el tercero, el estilo permisivo, se encuentra en padres con bajo nivel de control y alto nivel en responsabilidad; y el autoritario, se percibe en familias con alto nivel en control y bajo en responsabilidad.

Desde estas tipologías se sucedieron diversas investigaciones (Chao, 2001; Dornbusch, Ritter, Mont-Reynaud, Chen, 1987; Lamborn, Mounts, Steinberg y Dornbusch, 1991) que arrojaron luz a este ámbito, apuntando otras dimensiones de análisis del ejercicio del control familiar: coerción/imposición o aceptación/implicación (Musitu, Román y Gutiérrez, 1996). Los padres con altos niveles de implicación muestran cariño y afecto a su hijo cuando éste se comporta adecuadamente, y en el caso de que su conducta no sea la correcta, a juicio de los padres, tratan de dialogar y razonar con su hijo acerca de lo poco adecuado de su conducta. Por el contrario, los padres con bajos niveles de implicación/aceptación, suelen mostrar indiferencia ante las conductas adecuadas de sus hijos, es decir, no le muestran su agrado cuando éste se comporta de un modo correcto $\mathrm{y}$, de la misma manera, cuando su conducta es incorrecta tampoco razonan con él, ni le manifiestan sus opiniones y actitudes. Los padres se muestran realmente poco implicados con las conductas de sus hijos, tanto si éstas son correctas como si no lo son.

2 La reformulación de categorías se ha ido sucediendo, como lo encontramos en Ceballos y Rodrigo (1998) al clasificar los estilos educativos en democrático, autoritario, indulgente y negligente, o en Coloma (1993) al hablar de estilo autoritativo recíproco, para referirse a la autoridad compartida entre padres-hijos.

3 O autorizativo, según la traducción del inglés «authoritative». [El Diccionario de la Lengua Española, define autorizativo como «Que incluye o supone autoridad». Nota del editor]. 
Estas aproximaciones al estudio del estilo parental que han utilizado un enfoque tipológico o clasificatorio se completan con aportaciones que siguen un enfoque dimensional, de influencias múltiples (Palacios, 1999; Rivas, 2008). Desde esta perspectiva, se destacan dos supuestos fundamentales: a) las relaciones entre padres e hijos son bidireccionales, y b) las prácticas educativas sólo son eficaces si se adecuan a la edad de los hijos, y promocionan su desarrollo (Ceballos y Rodrigo, 1998). Desde dicho enfoque se analiza qué comportamientos observables en la familia pueden definirse, medirse formalmente y relacionarse directamente con el correcto desarrollo del niño (Amato y Fowler, 2002; Lee, Daniels y Kissinger, 2006; Prevatt, 2003).

\section{INVESTIGACIONES CENTRADAS EN LOS ESTILOS EDUCATIVOS}

Aunque la infancia es el período sobre el que preferentemente se han centrado los estudios, cada vez son más numerosos los realizados sobre la población adolescente. En ese caso, son ellos mismos quienes proporcionan la información sobre el estilo de sus padres, y, como han apuntado Gonzales, Cauce y Mason (1996), esta percepción suele mostrar menos sesgos y resultar más objetiva.

En términos generales, las investigaciones desarrolladas en contextos anglosajones y con población de clase media identifican el estilo autoritativo (que provee cercanía en la respuesta de los padres a la vez que mantiene un control firme con la madurez que requiere la situación) como el estilo educativo óptimo. A su vez, este estilo se asocia con el que ofrece mejor desarrollo en el adolescente en términos de competencia social, funcionamiento adaptativo, autoestima, autoconfianza y competencia académica (Carlson, Uppal y Prosser, 2000; Furnham y Cheng, 2000; Lamborn, Mounts, Steinberg y Dornbusch, 1991; Steinberg, Elmen y Mounts, 1989; Steinberg, Lamborn, Darling, Mounts y Dornbusch, 1994). De todos modos, existen estudios que señalan resultados opuestos en poblaciones distintas, aunque el número de estudios en otras culturas es menor. Así se señala que los adolescentes mexicanos (Villalobos, Cruz y Sánchez, 2004); filipinos (Hindin, 2005), alemanes (Wolfradt, Hempel y Miles, 2003), italianos (Marchetti, 1997) o brasileños (Martínez, Musitu, 
García y Camino, 2003; Martínez, García y Yubero, 2007) que reciben un estilo educativo permisivo obtienen un resultado similar que el de quienes describen a sus padres como autoritativos.

De igual modo, existen estudios que muestran resultados contradictorios al analizar el control en términos del grado en el que los padres demandan actuaciones a los hijos y tratan de dirigir sus comportamientos. En algunas ocasiones se asocian positivamente con el cariño y aceptación parental en adolescentes asiáticos (Rohner y Pettengill, 1985) y con lo contrario en las mismas culturas (Law, Lew, Hay, Cheung y Berndt, 1990), mientras que en las sociedades occidentales el control parental se asocia con hostilidad parental y rechazo. De ello se deriva la necesidad de analizar las relaciones y los estilos familiares de otras culturas orientales con mayor detenimiento.

\section{OBJETIVO DE ESTE ESTUDIO}

En el contexto de un amplio proyecto internacional que trata de conocer lo que los jóvenes sienten y opinan sobre las relaciones, el amor y la sexualidad en el que se enmarca este estudio (Proyecto YOUR LIFE) (de Irala et al., 2009), este trabajo trata de determinar qué estilo parental ?según la tipología de Maccoby y Martin (1983)?, se asocia con determinadas variables que tienen manifestaciones educativas en términos de competencia social (autoestima), competencia académica (altas calificaciones), funcionamiento adaptativo (impulsividad), autoconfianza (disposición a alcanzar metas planteadas), manifestación de formas de repulsa al sexismo y prevalencia en las relaciones sexuales, así como uso de las nuevas tecnologías y el empleo de su tiempo de ocio.

\section{METODOLOGÍA}

\section{INSTRUMENTO}

Para la obtención de los datos se elaboró un cuestionario compuesto por preguntas de diferente tipo, que analizaba cuestiones relativas a la vida de los adolescentes, a sus conocimientos y sus actitudes sobre cuestiones relacionadas con la afectividad, el amor y la sexualidad. El cuestio- 
nario, escrito en inglés coloquial, constó mayoritariamente de respuestas cerradas, y las preguntas actitudinales debían responderse en una escala Likert de 5 puntos.

Previamente se llevó a cabo un estudio piloto con 180 jóvenes, con el fin de asegurar la claridad y comprensibilidad de las preguntas planteadas, así como de adecuar el lenguaje a las características propias de la población filipina. Dicho cuestionario se elaboró para responderse en 45 minutos y de manera anónima.

\section{MUESTRA}

La muestra inicial a la que se dirigía el estudio era de 4000 estudiantes filipinos, con edades comprendidas entre 13 y 24 años de edad, que cursaran estudios de grado medio o superior. La muestra se obtuvo mediante un muestreo multietápico de universidades y colegios tanto públicos como privados.

Se seleccionaron 7 regiones de las 17 posibles de Filipinas: National Capital Region, Calabarzon, Central Luzon, Western Visayas, Central Visayas, Davao y Northern Mindanao, a partir de los siguientes criterios: 1) Elegir las regiones con mayor proporción de población adolescente y joven adulta. 2) Englobar cada uno de los tres grandes grupos de islas del país (Luzon, Visayas y Mindanao). 3) Abarcar una muestra lo más representativa posible de los diferentes estratos sociales y culturales del país. De este modo, en cada una de las 7 regiones se identificaron 4 instituciones: un instituto público, un colegio privado, una universidad estatal y una universidad privada. 4) Seleccionar los 4000 estudiantes en función del criterio de proporcionalidad de la muestra entre las 7 regiones elegidas.

El cuestionario fue contestado por un total de 3.726 adolescentes y jóvenes filipinos, lo que representó el 93\% de la muestra inicial de 4000 . De los cuestionarios entregados, se eliminaron del estudio el 3\% debido a alguna de las siguientes causas: no cumplir con el criterio de edad establecido en el estudio, $u$ omitir algún dato del cuestionario relevante para la investigación, como la edad o el sexo. Por tanto, la muestra definitiva final fue de 3.601 estudiantes, el 90\% de la población inicial. 


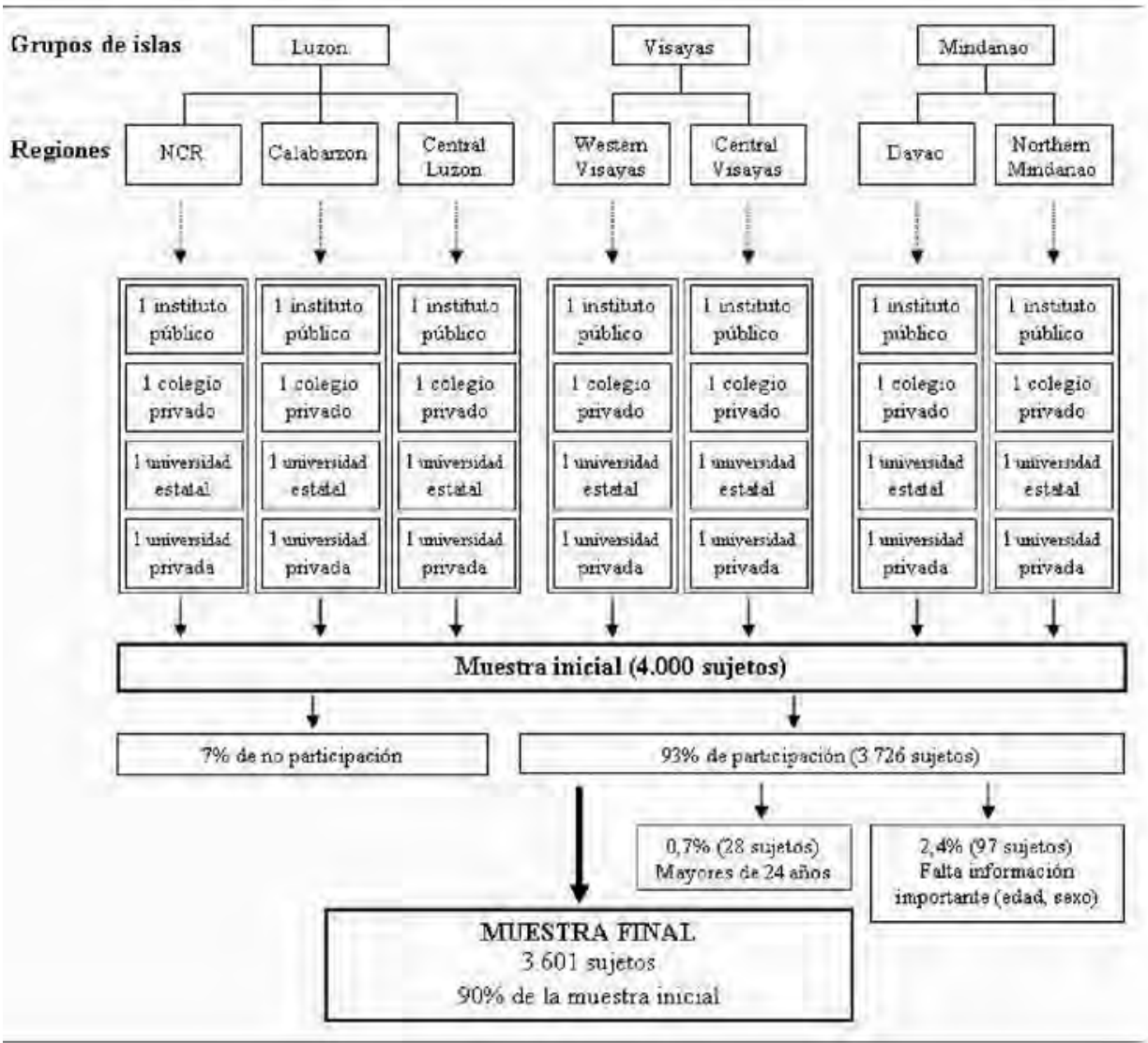

\section{RECOGIDA DE DATOS}

El cuestionario se respondió entre los meses de julio y septiembre de 2007, en 28 centros educativos de Filipinas, utilizando protocolos estandarizados de recogida de datos, previo consentimiento por parte de los centros educativos. 
Los encargados de recoger los datos de los cuestionarios se desplazaron a cada centro durante el horario lectivo. De este modo se aumentó la sensación de privacidad en los adolescentes al poder contestar las preguntas del cuestionario en el mismo centro, fuera del control paterno, y de forma voluntaria y anónima.

El procedimiento a seguir era el mismo en todos los centros educativos: en primer lugar, se procedía a la lectura de las instrucciones del cuestionario por parte de su administrador, recalcando el anonimato de los estudiantes a la hora de contestar a las preguntas, para un tratamiento más objetivo de la información recogida, y con la posibilidad de dejar preguntas sin responder. En segundo lugar, se otorgaba tiempo para responder a las preguntas del cuestionario durante el horario lectivo de los alumnos y con un tiempo limitado (45 minutos). En tercer lugar, los administradores procedían a recoger los cuestionarios, en sobres cerrados y sellados.

\section{VARIABLES}

El cuestionario contenía preguntas acerca de la reacción que manifiestan los padres ante un comportamiento negativo de los hijos. Los jóvenes debían responder con qué frecuencia reciben un castigo (justo o injusto) y/o una explicación por parte de los padres de por qué su comportamiento había sido reprobable. La escala para valorar dichas respuestas iba de 1 a 5 puntos (de «nunca» a «siempre»).

Sus respuestas se clasificaron de la siguiente manera:

1) Estilo autoritario: población que refiere castigo siempre o casi siempre, y que no se acompaña de explicación o de razonamiento de por qué motivo la conducta era inadecuada. Se incluyen también en esta categoría todos los que señalan recibir un castigo abusivo (con o sin explicación).

2) Estilo autoritativo: población que refiere castigo justo y que se acompaña de explicación o de razonamiento adecuado.

3) Estilo permisivo: población que refiere no recibir castigo, pero sí explicación.

4) Estilo negligente: población que refiere no recibir castigo, ni tampoco explicación. 
El cuestionario mide también la competencia social en términos de autoestima. La información del cuestionario se refiere tanto a la estimación de la capacidad personal (cognición) como al juicio de la valía personal (afecto) del alumno, componentes básicos de la autoestima. Las preguntas del cuestionario fueron: 1) «Creo que tengo bastantes buenas cualidades». 2) «Estoy satisfecho con mi apariencia física». 3) «Me siento querido por otras personas». 4) «Soy capaz de hacer feliz a otros».

Los alumnos debían contestar estas preguntas a partir de una escala con puntuaciones que iban de 1 «completamente en desacuerdo» a 5 «completamente de acuerdo». Para la investigación se valoró el sumatorio final de las puntuaciones (de 4 a 20 puntos; es decir, a mayor puntuación mayor autoestima).

De forma parecida el cuestionario incluyó preguntas acerca del componente adaptativo relativo a la impulsividad. A los alumnos se les plantearon tres cuestiones: 1) «Normalmente acabo las cosas/proyectos que he empezado» (puntuación inversa). 2) «Tiendo a querer/conseguir las cosas inmediatamente». 3) «Pienso y planifico el futuro incluso en cosas pequeñas» (puntuación inversa). Para la investigación se valoró la variable impulsividad en función del sumatorio final (de 3 a 15 puntos).

El cuestionario recabó información acerca de las calificaciones académicas medias, en una escala de 4 opciones (por debajo de 75, 75-79, 80-89 y 90 o más ${ }^{4}$ ). Se valoró la escala según dicha puntuación, y se calificaron como notas altas los que referían tener una media igual o superior a 80 .

El cuestionario también recogía información sobre las metas de personalidad planteadas por la población de estudio a través de 5 cuestiones relativas a posibles metas de futuro: 1) «Ser solidario». 2) «Ser leal». 3) «Ser sincero». 4) «Tener mi propia personalidad, de manera que mantenga mis opiniones».5) «No depender de las circunstancias». Debían contestar si estaban de acuerdo con las metas personales planteadas a partir de una escala de 1 a 5 (de 1 «nada» a 5 «mucho»), y se valoraron tanto de modo independiente como en función del sumatorio final de las cinco preguntas (escala de puntuación total de 5 a 25).

4 Notas sobre 100. 
En relación con la cuestión anterior, se preguntó en el cuestionario acerca de sus intenciones futuras referidas al matrimonio, a partir de 3 cuestiones: 1) «Elegir a la persona adecuada con quien formar una familia». 2) «Esperar a tener relaciones sexuales hasta el matrimonio». 3) «Si me caso, que mi matrimonio dure para toda la vida». Debían contestar las preguntas planteadas a partir de una escala de 1 a 5 (de 1 «nada» a 5 «mucho»), y se valoraron tanto de modo independiente como en función del sumatorio final de las cinco preguntas (escala de puntuación total de 3 a 15).

Además, respecto de cada una de estas 8 metas (de personalidad o referidas al matrimonio), también se les cuestionaba si los mensajes recibidos por parte de sus padres les ayudaban a alcanzarlas.

Por otro lado, el cuestionario recogía información sobre algunas variables sociológicas de la población, como el uso de las nuevas tecnologías, empleo de su tiempo de ocio, prevalencia de relaciones sexuales y opinión sobre cuestiones sexistas.

El cuestionario preguntaba acerca de cuántas de las siguientes condiciones se cumplían en las familias de la población analizada en cuanto al uso de las tecnologías de la información y la comunicación (TICs). Las preguntas fueron: 1) Tener en casa ordenador con acceso a Internet sin filtro. 2) Tener ordenador en el propio dormitorio. La respuesta positiva se valoró con 1 punto y la negativa con 0 . Se valoró esta variable a partir del sumatorio final de las dos preguntas (de 0 a 2 puntos).

Se evaluó paralelamente el estilo educativo familiar con la utilización del tiempo del ocio «no constructivo» de la población analizada. Las cuatro preguntas se referían a con qué frecuencia semanal los estudiantes realizan la siguiente actividad: 1) «Reunirse en la calle». 2) «Ir a discotecas, bares o lugares donde se consume alcohol». 3) «Ir a centros comerciales». 4) «Reunirse en un local privado sin supervisión de los padres o de otro tipo». La respuesta se valoró con 1 punto en el caso de que la frecuencia sea igual o superior a una vez por semana, y con 0 puntos en el caso de frecuencias inferiores. La variable se valoró a partir del sumatorio de la puntuación final dada a las cuatro preguntas (escala de puntuación total de 0 a 4 puntos). 
Asimismo se preguntaba a los alumnos si han mantenido alguna relación sexual hasta el momento.

En relación con lo anterior, se trataba de conocer sus respuestas acerca de la reprobación de mensajes sexistas por parte de los medios de comunicación. Las preguntas planteadas eran: 1) «No me gusta que los medios muestren mujeres como objetos sexuales». 2) «No me gusta que los medios muestren hombres como objetos sexuales». 3) «No me gusta que los medios asocien la feminidad con tener más relaciones sexuales». 4) «No me gusta que los medios asocien la masculinidad con tener más relaciones sexuales». Los alumnos contestaron en qué grado están de acuerdo con las afirmaciones, en una escala de 1 a 5 ( 1 completamente en desacuerdo, 5 completamente de acuerdo). Se seleccionaron aquellos que respondían con valoraciones superiores a 3 en las cuatro afirmaciones.

\section{ANÁLISIS DE LOS DATOS}

Se realizó un análisis univariante y multivariante de los datos. Los datos descriptivos univariantes se obtuvieron con análisis estándar de frecuencias y medias de variables categóricas y cuantitativas, respectivamente. Al comparar grupos, se utilizó el test de Chi-cuadrado y el test exacto de Fisher (para comparar proporciones), y el ANOVA (para comparar medias). En los resultados descriptivos, se realizaron análisis ponderados para asegurar la representatividad de la muestra con respecto al tipo de centro educativo del que proceden los jóvenes (colegio/ universidad, público/privado). En los análisis multivariantes, se elaboraron regresiones lineales múltiples y regresiones logísticas no condicionales. Todos los análisis se efectuaron con el paquete estadístico SPSS 15.0.

\section{RESULTADOS}

Sociológicamente la población se distribuyó del siguiente modo: el porcentaje mayor de la población son mujeres $(63,6 \%)$, de entre 16 y 18 años $(51,1 \%)$. Asimismo, la mayoría de los encuestados profesan la religión católica $(82,7 \%)$, proceden de familias de clase media $(78,1 \%)$ y estudian en centros públicos $(54,0 \%)$. 


\section{Tabla 1. Distribución de los encuestados según las principales variables demográficas.}

\begin{tabular}{|c|c|c|c|c|c|c|}
\hline \multirow[b]{3}{*}{ CARACTERÍSTICAS } & \multirow{2}{*}{\multicolumn{2}{|c|}{$\begin{array}{l}\text { Total } \\
(\mathrm{N}=3601)\end{array}$}} & \multirow{2}{*}{\multicolumn{2}{|c|}{$\begin{array}{l}\text { Varón } \\
\text { (N=1311) }\end{array}$}} & \multirow{2}{*}{\multicolumn{2}{|c|}{$\begin{array}{l}\text { Mujer } \\
(\mathrm{N}=2290)\end{array}$}} \\
\hline & & & & & & \\
\hline & $\mathrm{N}$ & $(\%)$ & $\mathrm{n}$ & $(\%)$ & $\mathrm{n}$ & $(\%)$ \\
\hline \multicolumn{7}{|l|}{ Sexo } \\
\hline Varón & 1311 & $(36,4)$ & & & & \\
\hline Mujer & 2290 & $(63,6)$ & & & & \\
\hline TOTAL & 3601 & $(100,0)$ & & & & \\
\hline \multicolumn{7}{|l|}{ Edad } \\
\hline $13-15$ & 1208 & $(33,5)$ & 450 & $(34,3)$ & 758 & $(33,1)$ \\
\hline $16-18$ & 1840 & $(51,1)$ & 639 & $(48,7)$ & 1201 & $(52,4)$ \\
\hline $19-21$ & 505 & $(14,0)$ & 200 & $(15,3)$ & 305 & $(13,3)$ \\
\hline $22-24$ & 48 & $(1,3)$ & 22 & $(1,7)$ & 26 & $(1,1)$ \\
\hline TOTAL & 3601 & $(100,0)$ & 1311 & $(100,0)$ & 2290 & $(100,0)$ \\
\hline \multicolumn{7}{|l|}{ Religión } \\
\hline Sin religión & 70 & $(1,9)$ & 36 & $(2,7)$ & 34 & $(1,5)$ \\
\hline Católica & 2977 & $(82,7)$ & 1079 & $(82,3)$ & 1898 & $(82,9)$ \\
\hline Protestante & 372 & $(10,3)$ & 135 & $(10,3)$ & 237 & $(10,3)$ \\
\hline Otras * & 182 & $(5,1)$ & 61 & $(4,7)$ & 121 & $(5,3)$ \\
\hline TOTAL & 3601 & $(100,0)$ & 1311 & $(100,0)$ & 2290 & $(100,0)$ \\
\hline \multicolumn{7}{|c|}{ Nivel económico ** } \\
\hline Bajo & 517 & $(14,4)$ & 214 & $(16,3)$ & 303 & $(13,2)$ \\
\hline Medio & 2813 & $(78,1)$ & 1009 & $(77,0)$ & 1804 & $(78,8)$ \\
\hline Alto & 271 & $(7,5)$ & 88 & $(6,7)$ & 183 & $(8,0)$ \\
\hline TOTAL & 3601 & $(100,0)$ & 1311 & $(100,0)$ & 2290 & $(100,0)$ \\
\hline \multicolumn{7}{|l|}{ Centro educativo } \\
\hline Público & 1944 & $(54,0)$ & 703 & $(53,6)$ & 1241 & $(54,2)$ \\
\hline Privado & 1657 & $(46,0)$ & 608 & $(46,4)$ & 1049 & $(45,8)$ \\
\hline TOTAL & 3601 & $(100,0)$ & 1311 & $(100,0)$ & 2290 & $(100,0)$ \\
\hline
\end{tabular}

* "Otras religiones» incluyen: judía, musulmana, hindú, budista, INK (Iglesia ni Kristo), aglipay, El $7^{\circ}$ día, pentecostal, mormona, testigos de Jehová, baptista, filipinista y "otra".

** Basada en la percepción del encuestado sobre el nivel económico de su familia. 
El estilo educativo más frecuente en la muestra encuestada fue el permisivo $(39,2 \%)$, seguido del estilo autoritativo $(29,1 \%)$. La diferenciación de los estilos educativos por sexos mostró que el estilo negligente es más frecuente en la población masculina, y el estilo autoritativo lo es en la femenina.

\section{Tabla 2. Estilos educativos.}

\begin{tabular}{|c|c|c|c|c|}
\hline & $\begin{array}{l}\text { Total } \\
\text { (N=3601) }\end{array}$ & $\begin{array}{l}\text { Varón } \\
\text { (N=1342) }\end{array}$ & $\begin{array}{l}\text { Mujer } \\
\text { (N=2259) }\end{array}$ & \\
\hline & $\mathrm{N}(\%)$ & $\mathrm{n}(\%)$ & $\mathrm{n}(\%)$ & $\mathrm{p}^{*}$ \\
\hline Autoritativo & $1048(29,1 \%)$ & $340(25,3 \%)$ & $708(31,4 \%)$ & $<0,001$ \\
\hline Negligente & $734(20,4 \%)$ & $321(23,9 \%)$ & $413(18,3 \%)$ & $<0,001$ \\
\hline Permisivo & $1413(39,2 \%)$ & $533(39,7 \%)$ & $880(39,0 \%)$ & 0,338 \\
\hline Autoritario & $406(11,3 \%)$ & $149(11,1 \%)$ & $257(11,4 \%)$ & 0,422 \\
\hline TOTAL & $3601(100,0 \%)$ & $1343(100,0 \%)$ & $2258(100,0 \%)$ & \\
\hline
\end{tabular}

* Valor p del test exacto de Fisher.

$\mathrm{Al}$ analizar la relación entre estilos educativos familiares y las variables estudiadas, el resultado señaló cómo en las familias filipinas con estilo autoritativo los hijos muestran mejores puntuaciones que en las familias que presentan estilos autoritarios, negligentes o permisivos. Así, dichos jóvenes tienen mayor autoestima y menor impulsividad, manifiestan mayor disposición a alcanzar las metas de personalidad y las relacionadas con el matrimonio, alcanzan con mayor frecuencia altas calificaciones escolares, presentan menor prevalencia de relaciones sexuales y desaprueban con mayor frecuencia las diferentes formas de sexismo. En estas mismas variables, las puntuaciones más bajas se obtuvieron en familias de jóvenes con un estilo educativo negligente. Las diferencias entre grupos fueron significativas en todos los casos $(\mathrm{p}=0,019$ para las relaciones sexuales, $\mathrm{p}<0,001$ en las demás variables). En cambio, no se apreciaron diferencias estadísticamente significativas en el uso inadecuado de las TICs ni en el ocio no constructivo. 
El resultado mostrado a través de un análisis multivariante (regresión lineal o regresión múltiple), ajustado por edad, sexo, religiosidad y nivel socioeconómico, señaló que los estilos educativos diferentes del autoritativo están asociados con peores puntuaciones en las variables autoestima, impulsividad, metas de personalidad, metas de matrimonio, altas calificaciones y desaprobación de mensajes sexistas. En cambio, las diferencias en cuanto a las relaciones sexuales dejaron de ser estadísticamente significativas.

Tabla 3. Asociación de diversas variables con el estilo educativo.

\begin{tabular}{|c|c|c|c|c|c|c|}
\hline \multicolumn{7}{|c|}{ ESTILO EDUCATIVO } \\
\hline & & Autoritativo & Negligente & Permisivo & Autoritario & $\mathbf{p}^{*}$ \\
\hline \multirow[t]{2}{*}{ Autoestima } & Media & 16,4 & 14,0 & 15,7 & 15,1 & $<0,001$ \\
\hline & $B(I C$ 95\%)** & (ref) & $-2,0(-2,3$ a $-1,6)$ & $-0,5(-0,8$ a $-0,2)$ & $-1,1(-1,5$ a $-0,7)$ & \\
\hline \multirow{2}{*}{ Impulsividad } & Media & 6,8 & 7,6 & 7,2 & 7,5 & $<0,001$ \\
\hline & $B(\text { IC 95\%) })^{* *}$ & (ref) & $0,7(0,5$ a 0,8$)$ & $0,3(0,2$ a 0,5$)$ & $0,6(0,4$ a 0,9$)$ & \\
\hline \multirow{2}{*}{$\begin{array}{l}\text { Metas } \\
\text { personalidad }\end{array}$} & Media & 23,5 & 21,6 & 22,6 & 22,7 & $<0,001$ \\
\hline & B (IC 95\%)** & (ref) & $-1,6(-1,8$ a $-1,3)$ & $-0,7(-0,9$ a $-0,5)$ & $-0,8(-1,1$ a $-0,5)$ & \\
\hline \multirow{2}{*}{$\begin{array}{l}\text { Metas } \\
\text { matrimonio }\end{array}$} & Media & 14,1 & 13,0 & 13,6 & 13,6 & $<0,001$ \\
\hline & $\mathrm{B}(\mathrm{IC}$ 95\%)** & (ref) & $-0,8(-1,0$ a $-0,6)$ & $-0,4(-0,5$ a $-0,2)$ & $-0,5(-0,7$ a $-0,2)$ & \\
\hline \multirow{2}{*}{$\begin{array}{l}\text { Uso negativo } \\
\text { TIC's }\end{array}$} & Media & 0,27 & 0,26 & 0,24 & 0,28 & 0,328 \\
\hline & $\mathrm{B}(\mathrm{IC} 95 \%)^{* *}$ & (ref) & $0,0(0,0$ a 0,1$)$ & $0,0(-0,1$ a 0,0$)$ & $0,0(0,0$ a 0,1$)$ & \\
\hline \multirow{2}{*}{$\begin{array}{l}\text { Ocio no } \\
\text { constructivo }\end{array}$} & Media & 0,96 & 0,92 & 0,97 & 1,03 & 0,359 \\
\hline & $\mathrm{B}(\mathrm{IC} 95 \%)^{* *}$ & (ref) & $0,0(-0,1$ a 0,1$)$ & $0,0(-0,1$ a 0,1$)$ & $0,1(0,0$ a 0,2$)$ & \\
\hline \multirow{2}{*}{ Notas altas } & $\mathrm{n}(\%)$ & $195(18,6 \%)$ & $63(8,6 \%)$ & $198(14,0 \%)$ & $63(15,5 \%)$ & $<0,001$ \\
\hline & OR (IC 95\%) $)^{* * *}$ & (ref) & $0,48(0,35$ a 0,65) & $0,76(0,61$ a 0,94$)$ & $0,82(0,6$ a 1,12$)$ & \\
\hline \multirow{2}{*}{$\begin{array}{l}\text { Relaciones } \\
\text { sexuales }\end{array}$} & $n(\%)$ & $132(13,2 \%)$ & $116(18,9 \%)$ & $214(16,7 \%)$ & $55(15,9 \%)$ & 0,019 \\
\hline & OR (IC 95\%) $)^{* * *}$ & (ref) & $1,28(0,95$ a 1,72) & $1,20(0,94$ a 1,55$)$ & $1,28(0,90$ a 1,83$)$ & \\
\hline \multirow{2}{*}{$\begin{array}{l}\text { Desaprueba } \\
\text { sexismo }\end{array}$} & $\mathrm{n}(\%)$ & $507(48,4 \%)$ & $182(24,8 \%)$ & $443(31,4 \%)$ & 158 (38,9\%) & $<0,001$ \\
\hline & OR (IC 95\%) $)^{* * *}$ & (ref) & $0,42(0,34$ a 0,52) & $0,53(0,45$ a 0,63) & $0,74(0,58$ a 0,94$)$ & \\
\hline
\end{tabular}

* Valor $p$ del test ANOVA (medias) o del test de chi-cuadrado (frecuencias).

* Coeficiente B (e intervalo de confianza al 95\%) en el modelo de regresión lineal ajustado por edad, sexo, religiosidad y nivel socioeconómico.

*** Odds Ratio (e intervalo de confianza al 95\%) en el modelo de regresión logística ajustado por edad, sexo, religiosidad y nivel socioeconómico. 
Si nos referimos en concreto a cada una de las metas de futuro, observamos en primer lugar que, en todas ellas, puntúan alto en mayor proporción aquellos alumnos cuyos padres les animan a alcanzar esas metas ( $p<0,001$ en todos los casos). A continuación hemos realizado un análisis multivariante para valorar la asociación entre la intención de alcanzar cada meta y las variables sobre los mensajes de los padres y el hecho de tener padres con estilo autoritativo. Este análisis se ajustó por edad, sexo, religiosidad y nivel socioeconómico. Se observó que tanto el mensaje de los padres como el estilo educativo autoritativo se asociaban con cada una de las metas de futuro analizadas, siendo todas estas asociaciones estadísticamente significativas (NC 95\%).

Tabla 4. Metas de futuro, mensajes de los padres y estilo educativo.

\begin{tabular}{|c|c|c|c|c|c|}
\hline & \multicolumn{3}{|c|}{ MENSAJE PADRES * } & \multicolumn{2}{|c|}{$\begin{array}{l}\text { MODELO MULTIVARIANTE } \\
\text { OR (IC 95\%)*** }\end{array}$} \\
\hline & $\begin{array}{l}\text { No } \\
\text { n (\%) }\end{array}$ & $\begin{array}{l}\text { Sí } \\
\text { n (\%) }\end{array}$ & $p^{* *}$ & $\underset{* * * *}{\text { Mensaje padres }}$ & $\begin{array}{l}\text { Estilo educativo } \\
\text { autoritativo } * * * * *\end{array}$ \\
\hline \multicolumn{6}{|l|}{ Metas personalidad } \\
\hline Ser solidario & $167(61,6 \%)$ & $2923(87,8 \%)$ & $<0,001$ & $3,81(2,92$ a 4,99$)$ & $1,49(1,18$ a 1,88$)$ \\
\hline Ser leal & $262(76,4 \%)$ & $2946(90,5 \%)$ & $<0,001$ & $2,56(1,93$ а 3,39$)$ & $2,32(1,71$ a 3,16$)$ \\
\hline Ser sincero & $260(71,0 \%)$ & $2949(91,2 \%)$ & $<0,001$ & $3,53(2,70$ a 4,61$)$ & $2,37(1,74$ a 3,24$)$ \\
\hline Mantener personalidad & $407(76,1 \%)$ & $2827(92,2 \%)$ & $<0,001$ & $3,10(2,42$ a 3,98) & $2,12(1,56$ a 2,88$)$ \\
\hline $\begin{array}{l}\text { Ser fuerte } \\
\text { ante dificultades }\end{array}$ & $276(69,3 \%)$ & $2978(93,0 \%)$ & $<0,001$ & $4,52(3,48$ a 5,88$)$ & $2,47(1,77$ а 3,45$)$ \\
\hline \multicolumn{6}{|l|}{ Metas matrimonio } \\
\hline $\begin{array}{l}\text { Elegir persona } \\
\text { adecuada }\end{array}$ & $462(78,8 \%)$ & $2817(93,5 \%)$ & $<0,001$ & $3,74(2,91$ a 4,80$)$ & $1,84(1,34$ a 2,52$)$ \\
\hline $\begin{array}{l}\text { No sexo hasta } \\
\text { el matrimonio }\end{array}$ & $350(38,8 \%)$ & $2290(84,8 \%)$ & $<0,001$ & $8,36(6,98$ a 10,01$)$ & $1,36(1,11$ a 1,66$)$ \\
\hline $\begin{array}{l}\text { Matrimonio para } \\
\text { toda la vida }\end{array}$ & $385(71,3 \%)$ & $2951(96,4 \%)$ & $<0,001$ & $9,14(6,93$ a 12,06$)$ & $2,48(1,64$ a 3,74$)$ \\
\hline
\end{tabular}

* Respuesta de los sujetos al ser preguntados si sus padres les ayudan a alcanzar cada una de las metas citadas.

* Valor $\mathrm{p}$ del test exacto de Fisher.

*** Odds Ratio (e intervalo de confianza al $95 \%$ ) ajustada por las variables «mensaje padres» y "estilo educativo autoritativo" y por edad, sexo, religiosidad y nivel socioeconómico.

**** Para cada meta, el modelo incluye el mensaje referido a esa meta concreta (y no los mensajes referidos a las otras metas).

***** En este caso se ha dicotomizado la variable, distinguiendo solamente las familias con estilo autoritativo de las que tienen otros estilos. 


\section{DISCUSIÓN}

En el contexto de un amplio proyecto internacional que busca conocer qué sienten y opinan los jóvenes sobre la afectividad, el amor y la sexualidad, el principal objetivo de esta investigación fue examinar el impacto del estilo parental percibido por los adolescentes filipinos en diversos aspectos de su ajuste psicosocial.

La realidad de los adolescentes filipinos muestra cómo, al igual que sucede en la mayoría de países occidentales, una educación autoritativa, que combina la disciplina moderada con la explicación razonada del motivo de la mala actuación, se asocia con distintos comportamientos que consideramos positivos en diversas áreas de su desarrollo. Así se mostró en el estudio en cuanto que los adolescentes que perciben a sus padres como autoritativos tienen un mejor ajuste psicológico y una mejor respuesta emocional (son menos impulsivos, obtienen mayor éxito académico, desaprueban con mayor frecuencia mensajes sexistas). De igual forma, parecen manifestar un sentido mayor de competencia (más intención de alcanzar en el futuro metas positivas), que la población con otros estilos educativos familiares.

En primer lugar, es interesante considerar la diferencia existente entre la población femenina y la masculina en los estilos negligentes y autoritativos. Mientras que el estilo negligente es más frecuente en la masculina, el estilo autoritativo lo es en la femenina. El hecho de que sean los propios adolescentes y jóvenes quienes proporcionen la información no nos permite indicar si son los padres quienes tienden a utilizar distintos estilos según el sexo de sus hijos, o si son los hijos quienes, en función de su sexo, tienen una diferente percepción de la autoridad de sus padres, aspecto que podría ser origen de investigaciones posteriores.

Una segunda conclusión que se extrajo de este estudio es el alto porcentaje de familias con un estilo educativo permisivo según la percepción de sus hijos, familias que sí dan explicaciones pero que no emplean mecanismos de control como puede ser el castigo, tan propio de culturas orientales (Lau, Lew, Hau, Cheung y Bendt, 2001). Resulta interesante encontrar este estilo en culturas orientales, puesto que en ellas existe gran interdependencia 
entre los miembros y el rol del castigo y de la disciplina familiar es una nota característica. Partiendo de la base de que los estilos educativos se enmarcan en una cultura determinada, en este caso la oriental, este estudio evidencia las grandes diferencias que separan a Filipinas respecto de otras culturas asiáticas corroborando lo señalado en otros estudios (Peabody, 1968, Chao y Tseng, 2002), y las grandes semejanzas con poblaciones de cultura occidental (Pittnam y Chase-Lansdale, 1991).

Es interesante señalar en tercer lugar que este estudio también corroboró lo analizado por otras investigaciones acerca de la relación entre el estilo educativo autoritativo y el alto rendimiento escolar. Diversos estudios han señalado que este estilo promueve mayor éxito que los demás en este ámbito (Dornbusch, Ritter, Leiderman, Roberts y Fraleigh, 1987; Steinberg, Elmen y Mounts, 1989).

Asimismo, este estudio manifestó que el estilo autoritativo es el que más fomenta la autoestima entre la población filipina, en principio porque se supone que las prácticas basadas en estilos comunicativos y de apoyo aumentan la capacidad de influencia y de control sobre los hijos, y la posibilidad de que adquieran y mantengan estilos educativos saludables.

En cuarto lugar podemos mencionar que la relación encontrada entre los estilos educativos de los padres y la actividad sexual de los jóvenes coincide con lo que indican otros estudios. Aunque no se tiene constancia de otras investigaciones que relacionen directamente la experiencia sexual con el estilo autoritativo, sí existen hallazgos que la relacionan con las dos características principales de dicho estilo: control y cercanía. Concretamente, por un lado, la investigación mostró consistentemente que el ejercicio de la autoridad parental disminuye la prevalencia de relaciones sexuales (Bersamin, 2008; Borawsky, 2003; Meschke, 2000). Por otro lado, esta prevalencia también es menor entre los jóvenes cuyos padres mantienen buena comunicación con ellos (Bersamin, 2008), tienen confianza en ellos (Borawsky, 2003) o los tratan con cercanía afectiva (Meschke, 2000). 
No disponemos, en cambio, de estudios con los qué comparar nuestros resultados sobre el sexismo. Era de esperar que un tipo de educación que reduce la probabilidad de tener relaciones sexuales aumente también el rechazo hacia diversas formas de sexismo, pero ésta es quizá la primera evidencia empírica directa de esta relación.

En cuanto a datos sociodemográficos podemos apuntar que la presente investigación corroboró la distribución de la población esperada en cuanto a religión profesada en Filipinas, puesto que, según la Oficina Nacional de Estadísticas de Filipinas (National Statistics Office, 2003), un $81 \%$ de los filipinos son católicos, y un 8,2\% pertenecen a otras religiones cristianas. Respecto a la distribución por sexos, instituciones oficiales informan de mayores tasas de escolarización entre las chicas (UNICEF, 2005; World Bank, 2007), lo cual también se corresponde con la mayor presencia femenina en nuestra muestra.

En cualquier caso, aunque este estudio ha dado un paso adelante en explorar la relación entre el estilo parental y el funcionamiento de la población adolescente filipina, sus resultados deben interpretarse con cierta cautela por sus dos limitaciones. En primer lugar, se trata de medidas basadas en la percepción de los adolescentes. Sería interesante, de cara al futuro, considerar la perspectiva de los padres en relación con sus estilos educativos. En segundo lugar, su naturaleza transversal. Este hecho nos impide sacar conclusiones definitivas sobre relaciones causales entre las variables. El hecho de que dos variables estén asociadas no indica que una de ellas sea causa de la otra, sino que caben varias explicaciones posibles: causalidad inversa, bidireccionalidad, o una tercera variable que sea causa común. Sin embargo, el hecho de que la asociación se mantenga tras ajustar por diversas variables, y la naturaleza de las variables estudiadas, sugiere una relación concreta: el estilo autoritativo parece favorecer algunos aspectos de los hijos.

A pesar de estas limitaciones, una fortaleza importante del estudio es el gran tamaño de la muestra. Este hecho facilitó una mayor potencia estadística para la obtención de resultados significativos. Además, la representatividad de la muestra otorgó validez externa al estudio, haciéndolo extrapolable (con las debidas cautelas) a la población de 
estudiantes filipinos de 13 a 24 años. Por último, el hecho de haber realizado análisis multivariantes, ajustados por las principales variables sociodemográficas, aumentó la calidad de los resultados.

Para concluir podemos señalar que el presente estudio puede beneficiar en primer lugar a los educadores y profesionales que trabajan con adolescentes en cuanto que ofrecen una visión de qué tipo de control y apoyo parental reciben los adolescentes. En segundo lugar, puede resultar útil a los padres como punto de reflexión, en cuanto que refleja la percepción que sobre ellos mismos tienen sus hijos sobre qué aspectos de sus prácticas y estilos educativos pueden ser mejorados.

En cualquier caso, estos resultados pueden ser la excusa y el motivo para que tanto padres como educadores unan esfuerzos para establecer vías de colaboración efectivas.

\section{BIBLIOGRAFÍA}

AMATO, P.R.; FOWLER, F. (2002). «Parenting practices, child adjustment and family diversity», Journal of Marriage and Family, 64, 703-716.

BAUMRIND, D. (1968). «Authoritarian vs. authoritative parental control», Adolescence, 3, 255-272.

BAUMRIND, D. (1971), «Current patterns of parental authority», Developmental Psychology Monographs, 4, part 2.

BERSAMIN, M.; TODD, M.; FISHER, D.A.; HILL, D.L.; GRUBE, J.W., \& WALKER, S. (2008), «Parenting practices and adolescent sexual behavior: A longitudinal study», Journal of Marriage and the Family, 70 (1), 97-112.

BORAWSKI, E.A.; IEVERS-LANDIS, C.E.; LOVEGREEN, L.D. \& TRAPL, E.S. (2003), «Parental monitoring, negotiated unsupervised time, and parental trust: The role of perceived parenting practices in adolescent health risk behaviors», Journal of Adolescent Health, 33 (2), 60-70. 
BRONFENBRENNER, U. (1987), La ecología del desarrollo humano, Barcelona, Paidós.

CARLSON, C.; UPPAL, S. \& PROSSER, E.C. (2000), «Ethnic differences in processes contributing to the self-esteem of early adolescent girls», Journal of Early Adolescence, 20, 44-68.

CEBALLOS, E. y RODRIGO, M.J. (1998), «Las metas y estrategias de socialización entre padres e hijos», en RODRIGO, M.J. y PALACIOS, J. (coords.), Familia y Desarrollo Humano, p. 225-243. Madrid, Alianza.

CHAO, R.K. (2001), «Extending research on the consequences of parenting style for Chinese Americans and European Americans», Child Development, 72, 1832-1843.

CHAO, R. \& TSENG, V. (2002), «Parenting of asians», 59- 94, in BERNSTEIN, M. (ed.), Handbook of parenting, Vol. 4, Social conditions and applied parenting, Lawrence Erlbaum Associates.

COLOMA, J. (1993), «Estilos educativos paternos», en QUINTANA, J. (coord.), Pedagogía Familiar, p. 45-58, Madrid, Narcea.

DE IRALA, J.; OSORIO, A.; LÓPEZ DEL BURGO, C.; BELEN, V.A.; DE GUZMAN, F.O.; CALATRAVA, M. et al. (2009), «Relationships, love and sexuality: what the Filipino teens think and feel», BMC Public Health, 9: 282.

DORNBUSCH, S.M.; RITTER, P.L.; LEIDERMAN, H.; ROBERTS, D.F. \& FRALEIGH, M.J. (1987), «The Relation of Parenting Style to Adolescent School Performance», Child Development, 58 (5), 1244-1257.

FULIGNI, A.J. (1998), «Authority, Autonomy, and Parent-Adolescent Conflict and Cohesion: A Study of Adolescents from Mexican, Chinese, Filipino, and European Backgrounds», Developmental Psychology, 34 (4), 782-792.

FURNHAM, A. \& CHENG, H. (2000), «Perceived parental behavior, self-esteem, and happiness», Social Psychiatry and Psychiatric Epidemiology, 35, 463-470.

GONZALES, N.A.; CAUCE, A.M. \& MASON, C.A. (1996), «Interobserver agreement in the assessment of parental behavior and parent-adolescent conflict: African-American mothers, daughters and independent observer», Child Development, 67, 1483-1498. 
HINDIN, M. J. (2005), «Family dynamics, gender differences and educational attainment in Filipino adolescents», Journal of Adolescence, 28, 299-316.

HUYNH, V.W. \& FULIGNI, A.J. (2008), «Ethnic Socialization and the Academic Adjustment of Adolescents from Mexican, Chinese, and European Backgrounds», Developmental Psychology, (44) 4, 1202-1208.

LAMBORN, S.D.; MOUNTS, N.S.; STEINBERG, L. \& DORNBUSCH, S.M. (1991), «Patterns of competence and adjustment among adolescents from authoritative, authoritarian, indulgent, and neglectful families», Child Development, 62, 1049-1065.

LAMBORN, S.D.; MOUNTS, N.S.; STEINBERG, L. \& DORNBUSCH, S.M. (1991), «Patterns of competence and adjustment among adolescents from authoritative, authoritarian, indulgent, and neglectful families», Child Development, 62, 1049-1065.

LAU, S.; LEW, W.J.F.; HAU, K.T.; CHEUNG, P.C. \& BENDT, T.J. (1990), «Relatinos among perceived parental control warmth, indulgence and family harmony of Chinese in mainland China», Developmental Psychology, 26, 674-677.

LEE, M.; DANIELS, H. \& KISSINGER, D.B. (2006), «Parental influences on adolescent adjustment: parenting styles versus parenting practices», The Family Journal: counselling and therapy for couples and families, 14, 3, 253-259.

MACCOBY, E.E. \& MARTÍN J.A. (1983), «Socialization in the context of the family: Parent-child interaction», 1-102, in E.M. HETHERINGTON (ed.), Handbook of child psychology, $4^{\mathrm{a}}$ ed., vol. 4, New York, Wiley.

MARCHETTI, B. (1997), Concetto di se'relazioni familiari e valori [Relations between family and value concepts], Unpublished Masters thesis, Universita degli Studi di Bologna, Italy.

MARTÍNEZ, I.; MUSITU, G.; GARCIA, J. F. y CAMINO, L. (2003), «Un análisis transcultural de los efectos de la socialización familiar en el autoconcepto: España y Brasil [A cross-cultural analysis of the effects of family socialization on self-concept: Spain and Brazil]», Psicologia, Educacao e Cultura, 7, 239-258. 
MARTINEZ, I., GARCÍA, J.F., \& YUBERO, S. (2007), «Parenting styles and adolescents' self-esteem in Brazil», Psychological Reports, 100, 731-745.

MESCHKE, L.L.; BARTHOLOMAE, S. \& ZENTALL, S.R. (2000), «Adolescent sexuality and parent-adolescent processes: Promoting healthy teen choices», Family Relations, 49 (2), 143-154.

MUSITU, G.; ROMÁN, J.M. y GARCÍA, E. (1988), Familia y educación. Prácticas educativas de los padres, socialización de los hijos, Madrid, Labor Universitaria.

MUSITU, G.; ROMÁN, J.M. y GUTIÉRREZ, M. (1996), Educación familiar y socialización de los hijos, Barcelona, Idea.

NATIONAL STATISTICS OFFICE (2003), 2000 Census of Population and Housing, in: [http://www.census.gov.ph/data/pressrelease/ 2003/pr0323tx.html].

PALACIOS, J. (1999), «La familia y su papel en el desarrollo afectivo y social», en F. LÓPEZ; I. ETXEBARRÍA; M.J. FUENTES y M.J. ORTIZ (coords.), Desarrollo afectivo y social, 267-284, Madrid, Pirámide.

PEABODY, D. (1968), «Group judgments in the Philippines: Evaluative and descriptive aspects», Journal of Personality and Social Psychology, 10 (3), 290-300.

PITTMAN, L.D. \& CHASE-LANSDALE, L. (2001), «African American adolescent girls in impoverished communities: The relationship between parenting style and adolescent functioning», Journal of Research on Adolescents, 11 (2), 199-224.

PREVATT, F. (2003), «The contribution of parenting practices in a risk and resiliency model of children's adjustment», British Journal of Developmental Psychology, 21, 469-480.

RIVAS, S. (2008), «Influencia bidireccional de los estilos parentales en la infancia», Journal of International and Developmental Psychology, 4, 65-74.

ROHNER, R.P. \& PETTENGILL, S.M. (1985), «Perceived parental acceptance-rejection and parental control among Korean adolescents», Child Development, 56, 524-528. 
ROUSSEAU, C.; HASSAN, G.; MEASSHAN, T.; MOREAU, N.; LASHLEY, M.; CASTRO, T.; BLAKE, C. \& MCKENZIE, G. (2009), «From the family universe to the outside world: Family relations, school attitude, and perception of racism in Caribbean and Filipino adolescents», Health and Place, 15 (3), 721-730.

SAMEROFF, A.J. \& CHANDLER, M.J. (1975), «Reproductive risk and the continuum of caretaking casualty» in F.D. HOROWITZ; M. HETHERINGTON; S. SCARR-SALAPATEK \& G. SIEGEL (eds.), Review of Child Development Research, vol. 4, 187-244, Chicago, University of Chicago Press.

STEINBERG, L., ELMEN, J. \& MOUNTS, N. (1989), «Authoritative parenting, psychosocial maturity, and academic success among adolescents», Child Development, 60, 1424-1436.

STEINBERG, L.; LAMBORN, S.D.; DARLING, N.; MOUNTS, N.S. \& DORNBUSCH, S.M. (1994), "Over-time changes in adjustment and competence among adolescents from authoritative, authoritarian, indulgent, and neglectful families», Child Development, 65, 754-770.

UNICEF (2005), The State Of The World's Children 2006. Excluded and invisible, in:

[http:/ / www.unicef.org/sowc06/pdfs/sowc06_fullreport.pdf].

VILLALOBOS, J.A.; CRUZ, A.V. y SANCHEZ, P.R. (2004), «Estilos parentales y desarrollo psicosocial en estudiantes de Bachillerato [Parental styles and psychosocial development in High-School students]», Revista Mexicana de Psicología, 21, 119-129.

WOLF, D.L. (1997), «Family secrets: transnational struggles among children of Filipino immigrants», Sociological Perspectives, 40 (3), 457-482.

WOLFRADT, U.; HEMPEL, S. \& MILES, J.N.V. (2003), «Perceived parenting styles, depersonalisation, anxiety and coping behaviour in adolescents», Personality and Individual Differences, 34, 521-532.

WORLD BANK (2007), Education at a Glance: Philippines, in: [http: / / siteresources.worldbank.org/EXTEDSTATS/ Resources/3232 763-1171296190619/3445877-1172014191219/PHL.xls]. 